\title{
Neue Beiträge zur Kenntnis einiger Wörter deutscher Abkunft.
}

Bei fr. 'huche, altfr. auch huge Kasten, Mehlkasten, von dem wohl auch sp. altp. hucha nebst bask. ucha stammt, verweist Diez auf das ml. hutica, das zu dem frz. Worte passe, wie ml. natica zu nfr. nache und altfr. nage, und fragt, ob hutica mit unserm deutschen hütte oder hotte zusammenhänge.

Aus ml. hutica kann das Wort nicht stammen, aber wohl darf dieses bei einer Herleitung von huge oder huche berücksichtigt werden. Neben ml. hutica finden wir bei DC als $\mathrm{ml}$. Nebenform hucha huchia, zu dem DC bemerkt, es sei eine vox Picardis nostris nota, wie er denn auch unter hutica angibt: est hutica, quod Belgae nostri huche vocant, cista major ac longior. Wir werden durch diese Angaben auf nd. Gebiet verwiesen. Das $t$ der Form hutica in Verbindung mit dem $k$-Laut der anderen ml. Formen und des frz. huche huge verlangt ein Wort, welches sowohl diesen, wie jenen Laut bot. Aus dem frz. huge oder huche hätte im Ml. nur entweder huc(h)ia huc(h)a oder hutia hucta, aber nicht ein hutica geschlossen werden können.

Im Ostfries. ist uns nun noch ein Wort erhalten, welches beiden Anforderungen entspricht, nämlich hukje, huktje, kleiner Koben, kleiner Winkel, kleine Ecke etc., ein Dim. zu ostfries. huk hok, welches „Loch, kleine, elende Wohnung, kleiner abgesperrter dunkler Raum oder Verschlag, Koben, Stall" etc. bedeutet, (das sich auch vielfach in 'Zusammensetzung erhalten hat, wie swîn-, hunde-, höner-, enter- huk), mnld. hock (ovile septum cors cavea), nld. hoc, wang. hek (Schafstall), das mit ostfries. hôk Spitze, Ecke, Winkel, Angel, mnld. hoek, mnd. hôk, huk und wohl auch mit nd. der hock, Grashaufen, schwed. der hock Haufe, die 'hucke Last, Bündel, zu der Wz. kuc biegen, krümmen, gehört. Ein Kompositum mäl-hukje oder huktje künnte zu der franz. Bedeutung Anlafs gegeben haben. Man vgl. auch ostfries. $h u k-s a k$, ein Brei aus gestampften Kartoffeln mit Mehl.

Juc frz. (jetzt veraltet) Hühnerstange, wall. joc, mit den Vb. frz. jucher sich auf die Stange setzen, um zu schlafen, pic. juquer, wall. joquer, neupr. s'ajoucá, engl. to juke, ist seiner Abkunft nach bisher unsicher. Nach Diez hätte mnld. hukken, mnd. huken, isl. hoka huka, schwed. norw. huka und dän. huge, welche zu dem eben 
angeführten huk gehören, und woher norm. hucher in dem Sinn von jucher stammt, einige Ansprüche, sofern man um des rom. Anlauts $j$ willen eine deutsche Zusammensetzung mit ge annehmen dürfe.

Baist (Ztschr. VI, 425) scheint hier das Richtige getroffen zu haben, wenn er der Form nach got. nld. juk, ahd. juh vergleicht, das im an. ok einen hölzernen Querbalken bedeute. Dirse selbe Bedeutung hat auch das ostfries. jük, Querholz zum Tragen von Lasten, und auch das ahd. juh zeigt sie, insofern es „Querholz zum Zusammenspannen zweier Zugstiere und Verbindungsglied zwischen 2 Brückenpfeilern oder 2 Bergspitzen" bedeutet. 1 Juc wird deshalb auf das Nld. zurückzuführen sein, während joug vom lat. jugum stammt.

Mets fr. Gericht, Speise, führt Diez, weil sich im Afr. auch mes findet, auf lat. missum in der Bedeutung "das Aufgetragene“, it. messo, zurück, indem er die Ableitung Wachters aus dem got. mats, ahd. maz, an. matr Speise, Mahlzeit, Nahrung, abweist. Die, wie er selbst zugiebt, alte Schreibung mets sei eine etymologische, um das Wort an das Vb. mettre zu knüpfen, nachdem das alte noch im subst. messe fortdauernde Partizip mes (mis) dem Sprachgefühle fremd geworden. Hätte Diez recht, so wäre auch noch anzuführen, dafs man die Form mets gewählt, um das Wort von anderen wie von mes = messager, meus und mais, afr. mes Haus, mes Richtung, mes-privé zu unterscheiden. Aber einmal erregt der Umstand Bedenken, dafs man schon in so früher Zeit etymologisch verfahren sein sollte, und zweitens scheint die älteste Form unseres Wortes mets selbst zu sein. Wenigstens bieten die Handschriften in Crestien, li contes del Graal met als casus obliquus an einer Stelle, wo Foerster erst dafür mit Unrecht mes gesetzt hat: $A$ chascun met don l'an servoit pardevant lui trespasser voit le graal, während gleich darauf als cas. obl. pl. les mes steht, bei dem sich der Ausfall der Muta wegen des folgenden $s$ erklärt.

Da mets sich mit $t$ nur auf französ. Gebiete zeigt, so werden wir auf das Nd. gewiesen, in dem sich das ahd. maz in einer dem fr. mets ganz nahe stehenden Form zeigt. Man vgl. mnd. nld. met, mnld. nfläm. met metle (pulpa suilla), afries. met(e) meit Speise, Fleisch, ostfries. met, as. meti mat, ags. aengl. mete maete. Die Form metz scheint sich näher an das got. mat-s, ahd. maz anzulehnen. Daneben zeigt sich noch eine andere Form mests. Sie dürfte einem erst aus got. mat $-s$ abgeleiteten deutschen Worte entsprungen sein, dem ahd. mast Mast, Mästung, Futter zum Mästen, eig. das Mit - Speisen - versorgt - werden, ostfries. mast Mast, Speise, Futter, Mästung etc., nd. mast, ags. aengl. maest.

Fat pr., fr. fade unschmackhaft, it. fado, pr. fr. fat, albern, thöricht, wird gewöhnlich vom lat. fatuus albern, hergeleitet, das poetisch im übertragenen Sinne auch „fade, unschmackhaft“ bei

1 Man vgl. frz. bau Querbalken zwischen beiden Seiten des Schiffes, vom nld. balk. 
Martial bedeutet. Das $u u$ soll sich in $u$ vereinfacht haben, wie im pr. zacs aus vacuus. Gaston Paris unterscheidet etymologisch zwischen fr. fat albern und fade unschmackhaft, und leitet nur das erstere aus dem lat. fatuus ab, weil im Fr. die Kombination uu, ua etc. den vorhergehenden Konsonanten schütze, so dafs sich das $t$ behauptet haben würde; fade selbst stamme vom lat. vapidus in der Bedeutung „verdorben, angerochen“. Hierzu will aber die Bedeutung nicht stimmen, denn vapidus bezeichnet nur „umgeschlagen, kahnig, verdorben", und was verdorben ist, schmeckt darum nicht "fade“, entwickelt vielmehr meist einen scharfen, unangenehmen Geschmack. Zudem kommt das Wort fade anscheinend auch noch nicht, worauf schon ten Doornkaat-Koolman (Wörterbuch der ostfries. Spr.) aufmerksam macht, im Afr. vor, so dafs schwer zu begreifen wäre, wie es nach Verlauf vieler Jahrhunderte aus vapidus entstanden und gebräuchlich geworden sein sollte. Es ist vielmehr wohl nd. Ursprungs, auf welchem Sprachgebiete der Stamm sich mehrfach findet, so im ostfries. fad fade fade, flau, ohne Saft und Kraft, mnld. vadde (laganum: libi admodum tenuis et flaccidi genus); vadde (fungus; clavus, patella in arboribus); vadde (ignava mulier); vadden (deflorere, flaccescere); vaddigh (flaccidus, languidus, ignavnis, torpidus); mfläm. vadde (bignet ou gepet); vadde (champignon d'arbres); vadde (femme faitarde); nnld. vadde vadelig vadze (träge, fahrlässige Person); vadzig träge, faul). Auch auf engl. Gebiet ist das Wort zahlreich vertreten, so als fadel schwach, matt, schal, fade, to fade welken, schwinden, faded schwach, abgelebt, fad Einfall, Grille, to fad läppisch sein, faddle spielen, tändeln, faddy läppisch.

Bague fr., Fingerring als Schmuckwerk, meist mit Edelstein, auch Ring, nach dem man rennt, wird von Diez auf das lat. bacca Perle, Gelenk der Kette, zurückgeführt, ebenso wie fr. baie Beere, pr. baga etc., - span. baya Hülse Schote, pg. baga.

Gegen diesc Ablcitung ist einmal cinzuwenden, dals lat. bacca zwar „Perle, Glied einer Kette“ (bei Prudentius) bedeuten kann, aber nur insofern als diese Gegenstände eben die Form einer bacca, einer runden Frucht, haben, so dafs man also nur dic einzelnen Glieder einer P'erlen-, Korallen-, oder Bernsteinkette damit bezeichnen könnte (s. Forcellini); aufserdem ist die letzte Bedeutung "Glied einer Kette" jedenfalls recht selten. Sodann bliebe es wunderbar, wenn bacca in diesem Sinne sich nur auf frz. Boden erhalten hätte. Damit ist der Vermutung Raum gegeben, dafs das Wort vielleicht einem dem französ. naheliegenden Gebiet entstammt. Und in der That finden wir in mehreren deutschen Dialekten entsprechende Wörter. So besteht im Ostfries. neben bogge in der Bedeutung „Bogen, Krümmung, Reif, Ring, Kranz" eine Nebenform bâge. Ebenso findet sich im As. bâggë̈bo Ringgeber (= ags. beággifa), bâgwini

1 Man vergleiche zum Anlaut altfries. faithe, engl. feud, das im mnld. veede anlautendes $v$ zeigt, u. a. Wörter. 
neben bogwini Ringfreund. Sollte eine ähnliche Bildung nicht dem fr. bague zu Grunde liegen?

Unsere Vermutung scheint bestätigt zu werden durch eine von Diez angeführte Bemerkung aus dem Gl. Isonis, wo es heirst: boja et baga unum sunt, wonach also boje (doch wohl das angeführte boge) und bage dasselbe wäre.

Das altfr. bou Armring, geht hingegen auf das ahd. boug, bouc etc., an. baugr zurück.

It. trovare, pr. cat. sp. trobar, fr. trouver finden, chw. truvar Recht sprechen, ein Urteil finden, sp. trovar dichten, trova Gedicht, hat bisher jeder Herleitung widerstanden. Grimm vermutete ein got. drupan $=$ ahd. trefan, wie trudan $=$ tretan, jedoch ist ein solches got. Wort nicht belegt.2 Von der Voraussetzung ausgehend, dafs sich in trovare augenscheinlich die Begriffe "finden und suchen oder holen" begegnen, wie denn „Dichten“, was der Provenzale mit trobar ausdrückt, ein Finden durch Suchen, durch Nachdenken und kein zufälliges Treffen sei, und sich auch in anderen Wörtern wie sp. pg. churw. parm. venez. catar "suchen“ und "finden“ berühre, will Diez es von lat. turbare durcheinander werfen, ableiten, woraus dann die Bedeutung „durchstöbern, durchsuchen“ ohne Schwierigkeit hätte erfolgen können. Dieselbe Begriffsentwicklung liege im it. frugare mit der Gabel durcheinander werfen, durchsuchen, und umgekehrt in rovistare durchsuchen und durcheinanderwerfen, vor. Die Formenveränderung turbare in trubare mache nicht das geringste Bedenken und jeden Zweifel müsse die Wahrnehmung beseitigen, dafs sich altpg. trovar in der Bedeutung turbare finde, wie neap. struvare $=$ disturbare, controvare = conturbare.

Wenn aber das genannte Verbum wirklich von turbare käme, so bliebe es doch immerhin wunderbar, dafs die roman. Sprachen alle ohne Ausnahme die Wandlung von turbare in trubare durchgemacht. Denn die eine vereinzelte Form torver, die sich in einem fr. Text aus dem Anfange des 12. Jahrh. findet, darf doch wohl nicht ins Gewicht fallen, und wenn auch anlautende Konsonanten, namentlich $t$ und $f$, ein $r$ gern an sich ziehen, (s. Diez, Rom. Gramm. I, 223) so ist doch diese Erscheinung in solcher Konsequenz in allen roman. Sprachen etwas sehr Seltenes und darf bei einem Worte, dessen Herleiturg noch so unsicher erscheint, nicht ins Gewicht fallen. Zudem sind wir in der Lage, ein Etymon zu bieten, bei dem eine solche Formenveränderung ausgeschlossen ist, bei dem man nur

1 Vgl. auch ostfries. ellbâg Ellenbogen, flitsbâg Armbrust, rägenbâg etc., bâgen, bôgen gekrümmt, bâgewid, bâgelbêr Bogenbier, Trinkgelage, sowie das wetterauische bâje beugen, schwed bagge $(\hat{a}=$ an. ahd. $\hat{a}, a s . \hat{a})$, mnd. bage = boge Bogen, mnd. bager Ring? (mit einem Edelstein?) bei Lübben.

2 Gaston Paris (Rom. VII, 748) stellt das Wort zum Kunstausdruck tröpus Sangweise, Melodie, woraus sich für trovare die Bedeutung komponieren, erfinden, entdecken, ergeben hätte; aber tropare und tropator sind in diesem liturgischen und musikalischen Sinne, wie schon Léon Gautier, (Monde 1873) nachweist, mehr als selten. 
eine ähnliche Bedeutungswandlung annehmen müfste, wie sie Diez für turbare in Anspruch nimmt. Dies ist das ahd. truopan truoban truaban trôban drôpan in Unruhe bringen, aufregen, verwirren, trüben, betrüben , 1 got. drôbjan erschüttern, aufregen, mnd. drôven, mnld. drocven (dolere, tristari, turbare), as. drôbhjan druovjan, ostfries. dröfen, dessen ursprüngliche Bedeutung „durcheinandermachen und rühren, verwirren" ist, wie bei dem lat. turbare. Ist die Herleitung des Verbums truoban mit den zugehörigen Adjektiven und Substantiven auch dunkel, so würde die sinnliche Bedeutung desselben der des rom. trovare ebenso gut, wie die des lat. turbare entsprechen.

Ebenso stimmen die roman. Formen des Verbums zu unserm truoban trôban; ich erinnere hinsichtlich derjenigen mit dem Vokal $o$ an it. fodero, sp. pg. forro, vom ahd. fuotar fôtar, got. fôdr, welches im Altfr. und Prov. mit Umwandlung des Vokals in der betonten Silbe fuerre, im fr. feurre, in den Ableitungen mit weiter gerücktem Ton fourrage fourrer etc. ergab, desgleichen an ahd. faldistôl, it. faldistorio, altfr. faudestueil faldestoel faldestoet faldestoed faudestuef, fr. fauteuil und ferner an ahd. alôd, it. sp. alodio, pr. alodi aloc alo, altfr. alued, fr. alleu. Man vergleiche mit den eben angeführten fr. und prov. Formen die betonten Formen des fr. u. prov. trouver, wie fr. troeve, trueve und später treuve, ferner truis etc., prov. truep truop. Während solche Diphthongierungen bei den Wörtern lat. Ursprungs sich nur aus kurzem $o$ ergaben, bilden die Wörter deutschen Ursprungs, wie schon Diez in seiner Grammatik ausspricht, und wie man aus den oben angeführten Beispielen ersieht, eine Ausnahme (vgl. auch Waltemath p. 76, 77), wohl weil das Ahd. eben den Diphthong uo neben langem $\hat{o}$ bot.

Altfr. gaignon wuignon, fr. gagnon, selten wagnon, ist ein früher ziemlich übliches, jetzt veraltetes Wort, dessen Herkunft nach Diez nicht frei von Zweifel ist. Es bedeutet wie fr. mâtin einen gewöhnlichen Haus- und Hofhund, Schäferhund, in Gegensatz zu levrier (= leporarius) Hasenhund, Windhund, altfrz. viaulre Jagdhund, it. segugio Spürhund, sigusius (in der Lex Sal. und Alam.), altfr. brachct Bracke. F.ben dazu gehört auch gagnier, das bei Rustebuef einen gemeinen Hund bezeichnet und oft von Menschen gebraucht wird, wie auch waignons selbst bei Jean de Condé im Chevalier a le mance Bd. I S. 218 Z. 1604 auf Sarazenen angewandt ist.2 Auch als Pferdenamen erscheint gaignon in Chanson de Roland, und wenn ich nicht irre, im Chanson des ducs de Normandie. Gaignon wird nun von Gachet für einen Spröfsling des auf den Ackerbau' an-

$1 \mathrm{Vgl}$. auch ahd. truobên trübe werden oder sein.

2 Auch sonst sind Beispiele der Anwendung von waignon auf Menschen, wie Herr Prof. Dr. Tobler mir mitzuteilen die Güte hatte, nicht selten. "Li mal waignon, li felon veautre" steht, von Juden gesagt, bei Barb. und Méon I, 285, 460; Seignors, cist dui gaignons, Qui ci vous requierent merci, Ont mort maint vassal, Claris 604 (von ruchlosen Raubrittern); Foie qui na comparison (die ewige Seligkeit) Lais por faire de toi waignon (zum Wucherer gesagt), Vers de le mort (herausg. v. Windahl, Lund 1887) Str. 153, 8. 
gewandten Wortes gaaing ${ }^{1}$ gehalten, wobei man an die Herkunft des synonymen it. mastino, fr. mâtin von it. masnada, altfr. maisnié Hausgenossenschaft, wie Diez angiebt, denken könnte. Der genannte Forscher will aber gagnon lieber auf canis, zu dem sich ein feminines cagne findet, gründen. Der Anlaut $w$, der sich in der Nebenform wagnon findet, ist aber an Stelle einer auf $c$ zurückweisenden Vorstufe $g$, wie Diez selbst erklärt, nur selten. Eben dieses $w$ bestimmt mich, nach einem deutschen Stammwort zu suchen, da ja Nebenformen mit anlautendem $w$ häufig auf deutschen Ursprung führen.

Im Deutschen findet sich eine Grundform vanga, die aus vorgerm. vanka entstanden ist. Sie liegt uns vor in got. vaggs $\pi \alpha \rho \dot{\alpha}-$ $\delta \varepsilon \iota \sigma o s$ liebliche, lustige Aue, as. wang, ags. vang, vong Aue, Gefild, altengl. engl. wang wong Feld, auch Aue, Ebene, Wiese, Niederung, altn. vangr, Feld, norw. dän. vang. Im Ahd. tritt sie auf in der Zusammensetzung holzwanga und holzwang = campus nemorosus, Aue im Holz, grüner blumiger Rasenplatz im Walde, und (wie auch im Mhd.) in zahlreichen Ortsnamen „zu den Feldern“. Ebenso zeigt sie sich noch im nhd. bair. und östreich. wang in der Bedeutung: Aue, grasiges blumiges Gefild in natürlicher lieblicher Vegetation ohne Hilfe menschlichen Anbaues, ferner im nhd. schweiz. wang wäng eine sich aufwärts ziehende Krinne an einem Felsen oder ein steiler Rain zwischen Gräben (s. Schade); auch das as. ahd. wang $\hat{a}$ die Wange, im Ostfries. auch so viel wie Fläche einer Mauer, gehört hierher, sowie der germ. lat. suevische Mannesname Vangio bei Tacitus Ann. 1 2, 29. 30 und der germ. lat. Name einer germanischen Völkerschaft Vangiones ${ }^{2}$ (im nachmaligen pagus Wormatiensis um den Donnersberg und östlich bis an den Rhein, im nördlichen Teile der heutigen bairischen Pfalz), deren Hauptort Borbetomagus (Worms) bei Ammianus und in der Notit. imp. Vangiones und noch später civitas Wangionum, Wangiona, Wangia genannt wird.

Sollte nicht dieses Wort wie der gleichgebildete Völkername und der angeführte Name der Stadt Worms unserm gagnon zu Grunde liegen, zumal wo gaignon Schäferhund bedeutet, der doch auf der Aue sein W'esen treibt? In gleicher Weise wäre es eine gute Benennung für ein Rols, das auf der Aue aufgewachsen ist. 3 Man vergleiche dazu das oben angeführte fr. mâlin, das zunächst nur den Haus- und Hofhund und erst in zweiter Linie einen Schäferhund bezeichnet. Wie sich hier die Bedeutungen vermischen, so auch bei gagnon, nur in umgekehrter Weise.

Liefse sich nachweisen, dafs der Stamm der obengenannten Völkerschaft sich noch länger erhalten hätte, so könnte man sogar an direkte Ableitung des Wortes aus Vangiones denken. Man vgl.

1 Vgl. it. guadagnare etc., altfr. gaagner das Feld bauen, erwerben, gewinnen, pr. gazanh, fr. gain Gewinn.

2 Caesar b. g. I,5 I etc., ein Kriegsvolk in den Reihen Ariovists = die in gesegneten Auen Wohnenden.

Vgl. das ähnlich gebildete champignon Feldschwamm. 
dazu sp. pg. galgo Windspiel (= canis gallicus, s. Ovid, Met. I, 533 : $U$ t canis in vacuo leporem cum Gallicus arvo Vidit, der mit keltischem Namen verlragus vertrahus (bei Martial 14, 200), im It. veltro, im Afr. ziaulre hei(st), und it. segugio, it. sp. alano, pg. alão, altfr. alan Dogge, Bullenbeifser (= alanus albanus, vgl. lat. molossus) sowie in anderer Weise fr. beruier, cagot etc. .

Fine andere Bezeichnung eines Hundes ist fr. baud, eine Art Windhund. Man leitet es, weil diese Tiere auch chiens muets genannt werden, auf gael. baoth taub, dumm, thöricht, got. bauth taub, stumm, zurück. Damit wird aber das fr. au und auch die Media nicht erklärt; $a u$ kann bei einem deutschen Worte nur aus $a$ vor $l$ mit folgendem Konsonanten, wie in fauve $=$ falwe, oder aus $a$ vor einfachem $l$ (ohne Analogie im lat - fr. Wortschatz) wie in fr. gaule Gerte, got. valus Stock, frk. *walu (s. Waltemath, Die fränk. Elem. i. d. franz. Sprache p. 85,86 ) entstehen. Es ist deshalb baud wohl auf ein anderes Etymon zurückzuführen. Liegt ein deutsches Wort zu Grunde, so entspricht, wie schon Littré gesehen, ahd. as. bald kühn, mutig, dreist, got. balths, ags. beald, bald kühn, tapfer, das im Mhd. auch die Bedeutung "schnell, eifrig" und ähnlich im mnd. bolt, ball, bald „rasch, kühn, trotzig"1 (vgl. auch das ahd. Adverb baldo kühn, mutig, dreist, schnell, sogleich) zeigt und auch sonst in die roman. Sprachen übergegangen ist als it. baldo, pr. baut baudos, altfr. baud keck, üppig, fröhlich, fr. baudet, altfr. baudouin Esel, etc.

Wie zu baudouin und baudet („das zufriedene vor Fröhlichkeit jauchzende Tier" Grimm, Reinh. pag. 244 in der Tierfabel) das germ. bald in der Bedeutung "fröhlich" in das Franz. Aufnahme fand, so zu baud Windhund, dasselbe Wort, aber in der Bedeutung "schnell", wie denn dieser Begriff der für einen Windhund bezeichnendste ist, wogegen der des "stummen“ zurücktritt, so dals er kaum bei der Bezeichnung einer Sppecies bestimmend gewesen sein kann.

It. stamberga bedeutet eine schlechte Hütte. Diez bemerkt zu dem Worte in aller Kürze, es sei nach seinem letzten Bestandteile zu schliefsen, ein deutsches Wort. Bugge (Rom. III, I63) stellt das Wort zum altfr. estamperche, welches unter ml. etarchartea bei DC nur in einer Urkunde erwähnt und mit pertica erecta erklärt wird, und meint, die ital. Behandlung des Wortes beruhe vielleicht auf Einwirkung des sinnverwandten aibergo, und was die Bedeutung anlange, so sei der Übergang von „Stange“ zu „Hütte“ nichts Ungewöhnliches. Diese Ansicht bestreitet Caix (Studi 59) und deutet stamberga als ein Mischwort aus stanza, welches im It. Aufenthalt, Wohnung, bedeuten kann (sp. estancia, pr. estansa Stellung, Lage, fr. étance Stütze), und albergo (Ztschr. I, 420). Aber beide Deutungen erscheinen als zu künstlich. Wozu sollte man die Kom-

1 Das ahd. bald ist nach Grimm mit lit. baltas weifs, hell, glänzend, verwandt; wir sehen hier einen ähnlichen Bedeutungswandel wie beim gr. á $\varrho \gamma o ́$ ' glänzend, schnell. 
position stanza + albergo gewählt haben, wo das einfache albergo genügt hätte? Auch entspricht albergo weder allein noch in einer Zusammensetzung mit stanza dem Begriff, da es nicht schlechthin eine schlech te Hütte, sondern zunächst, wie ich aus ml. Urkunden nachgewiesen zu haben glaube (s. Zeitschr. X), im Gegensatz zu herberge die berga, in der der Edle Aufnahme fand, und erst später "Wirtshaus" bezeichnet, ohne üblen Nebenbegriff.

Der zweite Bestandteil vom it. stamberga deutet unzweifelhaft, wie bei it. albergo, pr. alberga, fr. auberge, altfr. herberge, herbergier, fr. emberguer auf das noch im Deutschen vorliegende mhd. berge Herberge, und ahd. bërgan, got. bairgan umschliefsen, verbergen, und demgemäfs dürfte auch der erste Bestandteil zunächst in einem deutschen Worte $\mathrm{zu}$ suchen sein. Im Ahd. findet sich stein stain, got. stains = saxum, rupes, Fels, Stein, altn. steinn Fels, Stein, auch Felskluft und Steinhaus. Wie sich dieses Wort im Ahd. und Mhd. vielfach in Wortkompositionen vorfindet, so ist es auch in die roman. Sprachen übergegangen, so z. B. im it. stambecco, chw. stambuoch $=$ ahd. stainboch, welches im Afr. mit Umkehrung der Bestandteile bouc-estain ergab. Das deutsche $a i(e i)$ wurde im It. verschiedenfach $z \mathrm{u} a$, wie in $z a n a=$ ahd. zeina, got. tainjô, und im Fr. in betonter Silbe zu $a i$, wie im altfr. gaide, fr. guede, it. guado $=$ ahd. weit, fr. laid $=$ ahd. leid, got. [laids] etc.

Was die Bedeutung unseres Wortes anlangt, so würde es im eigentlichen Sinne eine "Steinberge“ d. h. eine Unterkunft, die in primitivster Art in einer Felsenkluft, einem Felsenloch sich befindet, oder „eine aus rohen zusammengeschichteten und nicht verbundenen Steinen hergestellte Unterkunft" bezeichnen. Analogien dazu bilden ahd. steinhol, Steinhöhle, Felsenloch, von dems. stein und ahd. hol, zu dem das mit bergan synonyme ahd. Verbum hëlan geheim halten, verhehlen, verbergen, gehört, ferner ahd. steinloh = foramen petrae, spelunca, gleichfalls eine Unterkunft primitivster Art.

Cahute fr., Baracke, altfr. chahute und cahuette, soll dem mnd. kaiute, nd. kajüte, schwed. kajuta, dän. kahyt, mfläm. kajute u. mnld. kaiute kaiuyte ihren Ursprung gegeben und aus diesen Dialekten wieder in der Form cajute Aufnahme in das Franz. gefunden haben.

Diez sagt von cahute, es habe das Aussehen einer Zusammensetzung. Ihr erster Bestandteil aber sei nicht ganz sicher, wahrscheinlich aber sei er das deutsche kaue, d. i. Käfig, Behältnis; der zweite sei offenbar das auch im Franz. vorhandene deutsche hütte und das ganze Wort bezeichne eine enge schlechte Hütte; cahuelte sei Diminutivform für cahutette und verhalte sich wie serviette zu servitette.

Was sich gegen diese Ableitung aus kaue sagen lärst, hat Diez selbst gethan. Deutsches $k$ ergäbe hier im Fr.ch und nur im Pikardischen $c$. Darnach müfste kaue im Franz. etwa $c(h) o e c(h) o u e$ gelautet haben, welches sich aber nicht nachweisen läfst. Man müfste deshalb annehmen, dafs das Kompositum schon im De utschen vorgelegen habe. 
Wenn wir die verschiedenen Formen, in denen unser Wort in den nd. Sprachen wiederkehrt, uns näher ansehen, so mufs es auffallen, dafs in diesen das $h$ des Fr. meist durch $i$ oder $j$ ersetzt zu sein scheint, welches dann auch im fr. cajute auftritt. Das dän. kahyt allein zeigt $h$. Wie ist dieser Wechsel zu erklären und wie wäre es zu begründen, dals auf dem am weitesten von Frankreich abliegenden Sprachgebiet, dem Dänischen, das fr. $h$ sich erhalten, während es in räumlich näher liegenden nd. Sprachgebieten durch $i$ oder $j$ ersetzt ist? Ist dies nicht ein deutlicher Hinweis darauf, dafs wir weder kaiute, cajute noch cahute (oder cahyt) als Urform ansehen dürfen, dals wir vielmehr nach einer Mittelform zu suchen haben, die beide Laute bot, aus der sich dann mit Unterdrückung des einen oder anderen Lautes jene oder diese Form ergeben mufste?

In dem zweiten Bestandteil liegt, wie fr. cahute und dän. kahyt zeigt und schon Diez bemerkt, wohl unzweifelhaft unser ahd. hutta, mhd. hütte, nd. hütt, nld. hut, mnld. hutte, norw. schwed. hytta, dän. hytte, (sp. huta), und so dürfen wir auch für den ersten Bestandteil ein deutsches Wort vermuten, wie denn das ch der altfr. Nebenform chahute unzweifelhaft auf deutsches $k$ weist, und zwar müfste es, wie ich oben geschlossen, ein Wort sein, dafs ein $i$ oder $j$ enthält. Was kann es nun anders sein als das in den nd. Mundarten bezeugte nd. mnd. kaje, mnld. kade kaeye, nld. kaai, schwed. kaj, dän. kai, ostfries. kâi kaje, wang. kôi. Es bezeichnet „die hölzerne oder steinerne Hafen- oder Ufereinfassung, bezw. den Damm oder das Bollwerk am Rande oder Ufer eines Hafenbeckens", und wird mit sp. cayo, altfr. caye Sandbank, pg. caes, fr. quai, ml. cayum Haus, die dem schon in den Isid. Glossen erwähnten kai (= cancellae), kaij (kaji? = cancelli, Schranken), entsprechen, aus kymr. cae Zaun, Umzäunung, bret. kaé auch Deich, kaéa einzäunen, hergeleitet. Schon ten Doornkaat-Koolmann hat in seinem Wörterbuche der ostfries. Sprache auf dieses kai kurz hingewiesen, ohne aber auf die Bedeutung des scheinbaren Wechsels von $h$ und $i$ Nachdruck zu legen, und sagt nur kurz: „Ist kajüte entweder mit kai (in der Bedeutung Schranke, bz. Verschluis) oder mit kau und koje verwandt, und kann auch altfr. cahuette (aus urspr. cantte mit eingeschobenem unorganischem $h$ ) ein Dimin. von kau sein?" Wir lösen mit obiger Deutung unseres Wortes die Schwierigkeit, die darin läge, dafs das altfr. Wort cahute, das selbst erst ein Lehnwort aus dem Deutschen ist, in das $\mathrm{Nd}$. übergegangen und dann wieder als cajute in das Fr. zurückgekehrt wäre.

Was die Bedeutung des Wortes selbst anbelangt, so bezeichnet es im Altfr. zunächst wohl eine rohe Hütte, die nur dazu dient, etwas zu verschliefsen (cf. oben die kymr. und bret. Wörter, sowie das Isid. kai), woher sich die Bedeutung des altfr. cahule Baracke erklärt; dann eine elende Hütte, die aus Brettern am Hafendamm oder auf dem im Hafen liegenden Schiffe hergestellt war, wie denn in früherer Zeit die Kajüte auf den älteren Schiffen nur ein kleiner 
abgezimmerter Raum, bz. ein kleiner Bretterverschlag war. Erst später bezeichnete man damit den Verschlag im Hinterraum des Schiffes, welcher zum Aufenthalt des Kapitäns oder eines anderen Schiffsoffiziers diente, und dann auf grölseren Schiffen als Personenraum für Passagiere benutzt wurde. In diesem Sinne hätte es dann zum zweiten Male in der Form cajute Aufnahme im Fr. gefunden.

Chiffe fr., schlechtes, dünnes Zeug, chiffon Lumpen, pik. chifer, fr. chiffonner, champ. chifouiller zerknüllen, piem. cifogn $=$ chiffe, etc., stellt Grandgagnage zu nld. kaf, mnd. kaf, kave, mhd. caf Spreu, während Diez sie, weil $e$ leichter zu $i$ wird als $a$, zum ahd. kevâ, das sich in Graffs Sprachschatz findet, ordnen möchte. Andere denken an arab. chiff, „vestis tenuis et pellucida“. Keine der angegebenen Ableitungen genügt aber. Mit den genannten Wörtern verbindet Diez einige sinnverwandte, die aber zum Teil nicht mehr üblich sind, wie chippe = chiffon, chipe, norm. chife Runken Brot, chipper in Stücke schneiden (= engl. chip), chipot Schnittchen, Kleinigkeit, chipoter sich mit Kleinigkeiten abgeben, chipault Lumpenkerl u. dgl. mehr.

Die zuletzt genannten Bildungen mit $p$ weisen auf einen deutschen Stamm, der noch vielfach im $\mathrm{Nd}$. auftritt. So erinnert chippe Lumpen und chipe Runken Brot, an das nld. kip Kerbe, Schnit t, Spalt bz. abgespaltenes Stück Holz, Kloben, Scheit, (Stück Holz am Pfluge), mnd. kip Zipfel, Spitze, nld. nd. mnd. kippe, kip Bund, Bündel, Packen, isl. kippa fasciculus etc., norw. kippe, ostfries. kippe kip Spitze, scharfe Kante (vgl. md. kippe Sichel, Art sichelförmiges Messer). Verbalbildungen dazu sind ostfries. kippen schneiden, hauen, kappen, spalten, sondern etc., mnld. mfläm. kippen greifen, fassen, schneiden, kerben etc. im Md., Nd. die Spitze abhauen, abschneiden, engl. chip in gleicher Bedeutung, an welches letztere schon Génin anknüpfte, aber ohne weitere Bildungen anzuführen, aengl. chippen, ags. cippjan secare, forcippjan praecidere, an. kippa schlagen, rücken, rucken, ziehen, isl. kippa raptare, in denen allen mehr oder minder die Bedeutung der roman. Bildungen zu Tage tritt. ${ }^{1}$

Dem fr. chipe Runken Brot, entspricht im Oberd. in der Bedeutung ein bei Rochholz in einer aargäuischen Sage erwähntes brolkipf; vgl. dazu auch das mhd. kipfe, bair. östr. kipfel kleines Weizenbrot. Auch sonst finden sich im Hd. entsprechende Bildungen wie kipfen in dem Sinne des nd. md. kippen, sowie mhd. kiffen nagen, beifsen, kauen, ahd. kipfa chifa chiffa, mhd. kipfe kipf ${ }^{2}$, allerdings nur in der Bedeutung "Runge, Stemmleiste am Wagen“. Wir dürfen aber auch hier von der Bedeutung "schneiden“ etc. ausgehen, da das nld. kip in gleicher Bedeutung sich findet, und für all die genannten Wörter auf gleichen Ursprung schliefsen. Die

1 Vgl. dazu auch die im Ablaut stehenden Bildungen wie nld. nhd. kappen vorn abschneiden, abhauen, nhd. oberd. koppen die Spitze abschneiden, kuppen cacuminare truncare, abkuppen.

* Auch Kluge läIst kipfa mit dem eben genannten kipfe verwandt sein. 
roman. Formen mit $f$ erscheinen ahd. Ursprungs, die mit $p$ lassen auf nd. Sprachstufe schliefsen.

Das fr. chipault Lump, mit der bekannten Endung ault, die eine schlimme Bedeutung verleiht, erinnert speziell an das nd. hd. kipper eine Person, die die Münzen beschnitt, Fälscher, Betrüger, (vgl. auch das mhd. kipper nicht rittermälsiger Kämpfer, ostfries. kipper in bôm-kipper der die Bäume beschneidet, hâr-kipper, $\hat{u} t$-kipper).

Gorra it. sp. pg., sp. auch gorro, bezeichnet eine Art Mütze. Die Grundbedeutung scheint nach Diez „Band“ oder „Binde“ gewesen zu sein, da das it. Wort auch „Weidenzweig", das pg. auch „Binsenstrick“ bedeutet und ein afr. gorre (bei Roquefort) mit ruban übersetzt wird. Die letzteren Bedeutungen scheinen es auszuschliefsen, dafs das Wort, wie Mahn meint, aus dem bask. gorria rot (eine Lieblingsfarbe für dieses Kleidungsstück bei den Basken) entstanden sei. Zudem zeigen die romanischen Formen kein $i$. Ebenso wenig vermag Baist's Erklärung, der das bask. Wort einem aus dem lat. burrus (= rufus) erst abgeleiteten unbezeugten burreus gleichstellen will, zu genügen.

Der Form und Bedeutung nach entspricht dem roman. Worte eine deutsche Bildung, wie das nd. gorre, welches sich neben gord, ostfries. gördel Gurt, Binde, Band, Riemen etc., überhaupt ein einund umfassendes etwas, Gurtriemen, Gurtband, Einfassung, Saumrand, findet und auch in dem abgeleiteten nd. Vb. gorren gürten, schnüren, binden etc. erhalten hat, während in den anderen Dialekten sich nur ein mit $d^{2}$ fortgebildeter Stamm zeigt. Dafs aber auch diese Bildungen mit $d$ einem kürzeren Stamme entsprungen sind, beweist das ags. on-gyran, ${ }^{3}$ das neben ongyrdan ungyrdan entgürten, entkleiden, auftritt; ebenso spricht dafür das dän. gyrtel, das nicht mit $d$, sondern mit $t$ fortgebildet ist, wie auch das engl. girt Gurt, sowie girt und girth gürten, bekleiden, neben gird. Neben den genannten Bildungen findet sich ein im Ablaut dazu stehendes ahd. garla kerlja Gerte, Rute, sceptrum, nld. ostfries. garde, mnd. gart garde Rute, Gerte, Zweig, gerde Rute, Stab etc., die wohl eine schlanke, biegsame, zum Binden geeignete Rute oder Weide (vgl. oben it. gorra in der Bedeutung „Weiden-zweig") bezeichnen, wie auch mhd. wî̀te, ahd. wîda, ags. vidhig, engl. wiihy (lat. vîte $x$ Abrahamsbaum, vîtis Ranke, gr. i $\tau \hat{\varepsilon} \alpha$ Weide, von Weiden geflochtener Schild, ǐvs dass.) ahd. wid widi Strick aus gedrehten

$1 \mathrm{Vgl}$. mnld. gorde (vinculum, lorum, cinctus, subligaculum) ags. gyrd, aengl. gurd, engl. girt, got. gairda Gürtel, an. gjördín etc. nebst ahd. gurtil(a), mnld. nld. gordel, ags. gyrdil gertel, etc. dän. gryrtel.

2 Vgl. noch ostfries. görden, mnd. gorden (cingere), as. gurdjan, ags. gyrdan zäumen, umzäumen, umschliefsen, einfassen, gürten, aengl. gurden, engl. girt, gird, girth gürten, umschliefsen, bekleiden etc., an. gyrda, ahd. gurten curten.

3 Vgl. die indog. Wz. ghar, skr. hur, zend. zar nehmen, fassen, greifen, die auch dem lat. hortus Garten, ahd. gart cart Gehege, Einfriedigung, garto Garten, zu Grunde liegt. 
Reisern, Geflecht aus Ruten etc. lat. vitta Binde, zu einem Stamme vith und vîth gehören, der im got. vidan, ahd. wëlan binden, skr. vițikâ Band, Binde etc. vorliegt.

Das altfr. gorre, Prunk, Schmuck, Falbel, das Scheler im Nachtrage zu Diez' Etymolog. Wörterbuch ebenso wie Godefroy mit dem altfr. gorres „rubans livrées des nouveaux mariés“ zusammenstellt, ist gleicher Herkunft. In den oben angeführten Wörtern trafen wir ja öfter den Begriff „Saumrand (s. ostfries. görde), Einfassung“, woraus sich die Bedeutung „Falbel,1 die zum Schmuck und Prunk dient", ergiebt. Und ebenso findet sich in den Verbalbildungen öfter die Bedeutung „bekleiden“.

Bei dem it. goffo, sp. gofo, fr. goffe plump, tölpelhaft, it. auch plump gearbeitet, erinnert Diez nur an das bair. goff Dummkopf, und meint, dies könne seinen Zusammenhang mit dem romanischen Worte nicht verleugnen. Derselbe Stamm wie in goff liegt aber auch sonst noch vielfach vor, worauf schon ten Doornkaat-Koolmann im Wörterbuch der ostfries. Sprache aufmerksam macht, so im isl. guffa, welches auch vappa bedeutet, schott. guff goff, engl. goff guff Dummkopf, Thor, närrischer Tölpel, Kauz, welches letztere schon Diez anführt, schott. guffie dumm, tölpelhaft, närrisch, ostfries. guffel alberner, närrischer Mensch, Narr, Thor, Dummkopf, auch lautes Lachen, geguffel anhaltendes lautes Lachen, engl. guffer Tölpel, Narr, ostfries. guff $\ln ^{2}$ anhaltend laut oder dumpf lachen, läppisch lachen. Diese Wörter sind augenscheinlich lautlich und begrifflich verwandt mit md. gûf Geschrei, mhd. güefen rufen, schreien, westfries. (in Hindelopen) goefe, ferner mhd. guft Geschrei, laute Freude, Uebermuth, Prahlerei, güften übermütig sein, prahlen, etc. guftic freudig, üppig, übermütig, guftlîch etc.

Daneben scheinen noch andre Stämme mit anderem Vokal und Auslaut im Deutschen vorzuliegen, wie denn hänfig im Auslaut einer deutschen Wurzel die zu einer Lautreihe gehörigen Konsonanten wechseln (s. Hildebrand, Einl. zum Buchstaben $k$ des Grimmschen Wörterbuchs). So im ostfries. nld. nd. gapen gaffen, gähnen, das Maul aufsperren, klaffen, offen sein etc., an. schwed. gapa das Maul aufsperren, gaffen, engl. gape (dän. gabe zeigt noch anderen Auslaut), ags. geapian, ostfries. gapp das Gähnen, Maulaufsperren, Müdigkeit, stupide Verwunderung, Ueberraschung; Gähnung, Oeffnung, Mund, an. gap Oeffnung, Schlund, Benehmen eines thörichten Menschen, schwed. gap weite Oeffnung, Schlund, aufgesperrtes Maul, Rachen, Loch, Chaos, (dän. gab Gebifs etc.) engl. gap Oeffnung, Spalte, Kluft, Gähnen, der Gähnlaut, ags. geap ge-

1 Vgl. sp. randa, pg. renda Rand, dann Spitze an Kleidern, das vom deutschen rand, ahd. rant, mnld. rand (margo, ora, crepido, lymbus) stammt, sowie nld. kant Rand, Spitzen.

2 Vgl. ostfries. gaffeln, gabbeln, mit weit geöffnetem Munde hell und laut lachen, sich in alberner Weise lustig machen, spotten, nd. gaffeln, dass., gaffen laut bellen, kläffen, engl. gaffle aufziehen, verspotten, belästigen. 
räumig. Im Ablaut dazu steht wieder as. gipên offen stehen, klaffen, mhd. giffen gaffen, mit offenem Maule gucken.

Bisweilen ist nicht genau festzustellen, ob ein urgermanisches $p$ oder $f$ (indog. $b$ oder $p$ ) im Stamme vorliegt, so beim md. mhd. mhd. gaffen, (neben md. gappen) mhd. gaffôn, mhd. giffen.

Das gewöhnliche mhd. ahd. Wort für unser "gaffen", das erst in nhd. Zeit wieder in den Vordergrund trat, ist das ahd. chapfên, mhd. bair. kapfen, ${ }^{1}$ gaffen, verwundert auf etwas schauen, das sich auch im Nd. findet als kapen, also mit anderem Anlaut. Daneben kommt im Mnd. auch japen, jappen, engl. jap in gleicher Bedeutung vor, wie sich denn bei Fick die Wurzeln gap, gabh jabh und zaf (zend. zafan Mund, zafare Rachen, Schlund, Hölle, zafra Mund, Rachen) finden.

Wie man auch über die Verwandtschaft dieser Wörter denken mag, eins steht fest, dafs die Bedeutung der zu Grunde liegenden Wurzel etwa "gähnen, den Mund aufsperren" ist, und aus dieser Bedeutung geht die der stummen oder der lauten Verwunderung, der stupiden Verwunderung, hervor, woraus sich wieder als Bezeichnung einer Person die eines albernen, dummen, tölpelhaften Menschen in den roman. und deutschen Bildungen ergiebt.

Auf Grund des mhd. guft güefen, md. gûf setzt nun ten Doornkaat-Koolman auch für das Ahd. ein gufan schreien, rufen, voraus, und zu diesem stellt er das it. gufo Ohreule, während Diez dieses Wort aus dem ahd. hûf hûvo mit Vertauschung des gutturalen Anlauts, wie sie noch bei venez. trient. garbo bitter, und neap. guoffola Wange, im Anlaut vorliegen soll, ableiten möchte. Was gegen die Ableitung, die Diez gibt, spricht, ist der Umstand, dafs im Ahd. nur hûrvo huvo hûo Eule, Uhu (mhd. hûwe, as. hûo) und nur einmal in den Emeraner Glossen mit $f$ im Auslaut huf, das dem it. gufo auch nicht entspricht, vorkommt. Diez scheint hier hîwo mit ahd. uifo ûvo, mhd. ufe, 2 auf Nachteule, verwechselt zu haben. Aber auch ten Doornkaat's Ableitung genügt, da das it. $u$ ein langes $\hat{u}$ voraussetzt, nur dann, wenn man von einer Form wie md. gîf (vgl. das hindelopisch - westfries. goefjen, gesprochen gîfjen ein surrendes dem Tone $h u-h u-h u$ entsprechendes Geräusch machen) ausgeht.

Auch in dem genannten ven. trient. garbo, com. garb gherb, cimbr. gerbe (it. garbelto Veneroni) bitter, ist wohl keine Ableitung aus dem mhd. har (e) her (e) (flect. harewer) herb, amhd. harewen exasperare, zu sehen. Es scheint von einem mit $g$ anlautenden Worte zu stammen, das sich auch sonst im Deutschen, wenngleich zu-

1 Vgl. auch mhd. kapfaere der verwundert schaut, Gaffer, ahd. kapf Ort, von dem man ausschaut, salzb. kapf vorspringendes Dachfenster, mhd. kaphspil, md. kaffespil Schauspiel, sowie unser kaffer, schles. laus. kaffer küffer Oeffnung, Loch, Dachfenster, kaffern klaffen, offenstehen, ostpr. kapfenster etc.

2 Vgl. auch das lat. bubo, (b a u b a ri kläffen), gr. $\beta \dot{v} \alpha \varsigma$ Uhu, $\beta \tilde{v} \zeta \alpha$ Eule, nd. pucken, ahd. phûchôn mit anderem Anlaut, sowie lit. yva Uhu. 
nächst nur als Substantiv (bisher unbekannter Herkunft) nachweisen läIst, ich meine das ahd. garawa garuwa garwa, mhd. garwe garbe, ags. gearewe, nld. gerw, siebenb. guor (auch in der Form grab in schabgrab) Garbe, Schafgarbe. Diese Pflanze enthält ein ätherisches Oel und einen bitteren Extraktivstoff (vgl. bittere garb bei Nemnich, s. Grimm), der deswegen gegen Krankheiten der Verdauungsorgane seit Alters her (s. Leunis) gebraucht wird, und ist also wohl nach ihrer Bitterkeit benannt. Mit diesem Worte scheint das schwache ahd. $\mathrm{Vb}$. garawen garwen gerben, dann gar machen, bereit und fertig machen, verwandt, in dem zum Teile noch die gleiche Bedeutung hindurchleuchtet. Garawen bezeichnet zunächst wohl weiter nichts als „bitter machen“. Denn das Gerben erfolgt durch Anwendung der bitteren Eichenlohe, wodurch das Leder in sich zusammengezogen und gefestigt wird. Das Verbum gehört ohne Zweifel (s. Schade) zu der indog. Wz. ghar, erweitert ghrbh und gharbh, ${ }^{1}$ greifen, fassen, an- und zusammenziehen. Die Bedeutung "bitter" ist wohl eigentlich aus der andern „zusammenziehend" (vom Geschmack etc.) hervorgegangen, ähnlich wie bei ahd. raffi asper, fr. mdartl. raffe herbe, sauer, com. rap asper, das zu dem im nd. rapen, nhd. raffen an sich reifsen, vorliegenden Stamme gehört, und wie unser her $b^{1}$ selbst sowie ahd. herbist Herbst, Erntezeit, mit gr.xa $\alpha \pi i \zeta \varepsilon \imath v$ ernten, $x \varepsilon i \varrho \varepsilon \ell v$ schneiden, $x \alpha \varrho \pi$ ó Frucht, lat. carpere pflücken, ahd.

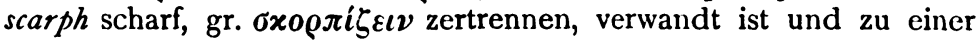
Wurzel karbh gehört.

Bei garawen entwickelte sich aus der Bedeutung „zusammenziehen (durch Anwendung von bitterer Eichenlohe)" dann die von „bereit und fertig machen, gar machen, rüsten“. Aehnliche Bedeutungsentwicklung dürfen wir auch einer kürzeren Bildung, dem ahd. garo garawo mhd. gar(e) garwe bereit, gerüstet, vollständig, gänzlich, ags. gearo gemacht, vollendet, fertig, gearo gearve ganz und gar, as. garu bereit, gerüstet, ${ }^{2}$ fertig, nd. gar, nld. ostfries. gâr gar (nicht roh), fertig, gegerbt, gekocht, reif, zusprechen, und es crscheint nicht ausgeschlossen, dafs auch diese die Bedeutung ,zusammenziehend, bitter" gehabt hat, und dafs speziell auf diese Bildung das it. garbo zurückgeht.

Aehnlich wird auch das neapol. guoffola Wange, das Diez zum ahd. hiufila Backe, Wange, ${ }^{3}$ stellen möchte, noch scine Lösung finden, wenn auch ten Doornkaat - Koolman's Herleitung aus dem ahd. goffâ gophî, mhd. goffe, md. guffe (clunis), das allerdings ähnliche Bedeutung zeigt, nicht genügen kann, da die Nebenform

1 Daneben existiert noch eine indog. Wz. grbh, der eine Nebenform zu garbe, näml. karbe Feldkümmel (karbekraut auch Schafgarbe) zu entsprechen scheint.

2 Zum sbst. ahd. garawî Zubereitung, Zurüstung, Gewand, vgl. ahd. saro (sarawes) Rüstung, das mit ahd. sarf scharf, acer, acerbus, berlinisch sarb (sarbe kirschen), ahd. serfan exasperare, lat. sarpere beschneiden, gr. $\alpha \varrho \pi \eta$ verwandt erscheint.

3 Vgl. auch ahd. huf Hüfte, das eines Stammes mit hiufila ist. 
vuoffola auf deutsches anlautendes $w$ schliefsen lälst. Vielleicht liegt hier eine Ableitung aus dem deutschen Stamme vip vor, der im ahd. wîfan, nd. nld. wippen windend hin- und herdrehen, schwingen, und seiner Sippe entwickelt ist. Man vgl. zur Bedeutung das ahd. wang $\hat{a}$, it. guancia, das zu einer Wurzel vang sich drehen, sich bogenförmig bewegen (vgl. ostfries. wingern sich hin- und herbewegen, wenden, drehen), gehört.

Urlare it., sardin. urulare, wal. urld, fr. 'hurler ist man geneigt, aus dem lat. ululare abzuleiten, und beruft sich, um den Wandel in den Liquiden wahrscheinlich zu machen, auf it. zirlare, sp. chirlar, chirriar, pg. chirlar chilrar schreien, zwitschern, die auf ein aus zinzilulare ${ }^{1}$ verkürztes, angenommenes zilulare zurückgeführt werden. Beide Ableitungen erscheinen aber zu gewaltsam, als dafs sie Beifall finden könnten, wenn sich ein anderes Stammwort finden läfst. Besonders schwer fällt es dabei, den Anlaut des fr. 'hurler und auch den der it. Nebenform chiurlare zu erklären. Schon Schneller setzt deshalb für das letztgenannte Wort ein sbst. chiurla Eule, voraus, das er aus deutschem quirl entstehen lassen möchte, "weil die Eule beim Vogelfang auf einen Pflock gestellt wird und auf einem Fufse stehend beim Anziehen der Schnur sich dreht". Aber auch diese Erklärung erscheint zu gewunden.

Dem it. urlare entspricht genau ein germanisches Wort, wie das ostfries. hurreln ${ }^{2}$ (auch hurdeln, woran speziell pr. udolar anklingt), brausen, sausen, wirbeln, in wiederholten Stölsen stark wehen, engl. hurl strudeln, wirbeln, heulen, oberd. hurlen rollen, kollern, bair. frk. hurlen, horlen (auch hörgeln, schwäbisch hurglen, horglen, auch kurglen wälzen), schweiz. hurrlen mit dem Brummkreisel spielen, mhd. hurlebûs Lärmen, Tumult, Saus und Braus. Hurreln selbst ist der Form nach ein Iterativ zu mhd. nhd. hurren sich schnell, z. B. sausend und schwirrend bewegen (= lat. currere nach der Lautverschiebung) schwed. hurra schwirren, (vgl. noch nhd. hurri Zusammenstofs, Zank, Streit, engl. hurly Tumult), und gehört nach Bopp zu einer Wurzel kur (sonare).

Neben fr. 'hurler besteht ein fr. huler, uler, bei dem schon Diez Einmischung des deutschen heulen, ahd. hizvilôn laut rufen(? bei Otfried 5, 23, 22), mhd. hiuweln hiulen anzunehmen geneigt ist. Das Wort stammt aber wohl von einer md. mittelrh. ostfries. Nebenform hâlen heulen, laut schreien, dumpf tönen, rauschen; hulotte Eule, wird dem entsprechend von einer dem ahd. hiuwela hîwela, mhd. hiưvel hûwel entsprechenden Nebenform *hâl (a) (vgl. mnld. huile, sowie $\mathrm{nnld}$. uil $=$ ahd. ûzvila ûla, mhd. iuzvel iule) herzuleiten sein.

Und was das pg. huivar anbelangt, so gehört es wohl zum ahd. hîwwo hûo, mhd. hûzwe Eule, Uhu, oder speziell zu einer Neben-

1 Das barbarisch-lat. zinzilulare zwitschern, entstammt augenscheinlich einem ahd. Worte, das man nach dem mnld. tintelen (tinnire, klingen, schreien, zwitschern) vermuten darf.

' Ostfries. hurrel, hurdel Windsbraut, sausender Windstofs, kurzdavernder Lärm, nld. horrel Stofs, Puff, Zank. 
form huizve, 1 wie wir ja auch neben ahd. hûwela im Mnld. huile fanden.

Chiurlare hingegen stellt sich unzweifelhaft, ebenso wie urlare zu hur reln, zu einem deutschen kurreln, das im Ostfries. „gurgeln, kollern, ein wiederholtes dumpfes Geräusch machen“, im Nd. „sich wälzen, rollen, ein Geräusch wie rollende Dinge machen, rappelig, toll sein" etc. bz. „toben, schelten, murren“ etc. bedeutet. Man vgl. dazu auch mnd. nd. kurren brummen, murren, mnld. koeren seufzen, mhd. nhd. kurren (stridere etc.), schott. curr gurren, an. isl. kurra gurren, murren, brummen, sausen.

Auf ein im Ablaut dazu stehendes Wort führt uns das oben genannte sp. pg. chirlar. Zwar ist hier im Deutschen nicht mehr eine Interativbildung nachzuweisen, aber das deutsche kirren hat dieselbe Bedeutung wie kurren stridere, crepare, murren, brummen, knirschen, schreien vor Angst, Hunger. Vgl. auch nd. kirren vom Schreien der Hühner, wenn sie einen Raubvogel sehen, kärnt. kirren kîren gellend schreien, heftig weinen, sowie ahd. chërran, mhd. kërren stridere, strepere, fremere, gellend schreien, klingen, und ahd. charrên stridere, isl. karra.

Und was das it. zirlare anbelangt, so dürfte es ebenfalls deutschen Ursprungs sein. Es setzt ein ahd. zirlen voraus, das sich im Ostfries. als tirreln tireln tirlen sich rasch und wiederholt hinund herbewegen, sich drehen, schwenken, wirbeln, schwirren, satl. tirlje, drehen, schwenken, dän. trilie sich drehen, rollen, erhalten hat.2

Das fr. guichet früher auch viquet, afr. wiket, kleinere Thür in einer grölseren, altfr. wiket, leitet Diez vom altn. vik Schlupfwinkel (auch Behältnis zum Verwahren), ags. vic ${ }^{3}$, (recessus, portus, sinus, angulus) ab. Es wären hier noch zu erwähnen ostfries. wîk wike das Weichen, Entweichung, Flucht, Zuflucht, ein von einem Hauptkanal seitlich abweichender Kanal oder überhaupt eine seitliche Ausbuchtung eines gröfseren Tiefes oder Kanals, Bucht, Meeresbucht, mnd. wîk Bucht, Weichen, Entweichung, mnld. wijck (perfugium, flexio etc. Sicherheitsort), aengl. wike, engl. wick Bucht, Winkel, Ecke, schwed. vik nebst mhd. wich, norw. vik kleiner Winkel, Krümmung, Biegung, kleine Abweichung und Veränderung etc. vik veek, schwed. veck Falte, Knick, Bruch, bz. Höhlung, Biegung, die alle zu dem im ahd. wîchan nicht fest stehen, bei Seite treten, sich zurückziehen, nachgebend Raum geben, enthaltenen Stamme gehören. Darnach kann es keinem Zweifel unterliegen, dafs Diez' Bemerkung, das engl. wicket, nld. winket stamme aus dem Romanischen,

$1 \mathrm{Vgl}$. auch nhd. huien schnell thun.

2 Vgl. auch ostfries. tir( $r)$ el tirl ein rasch hin- und her- und im Kreise drehendes, schwirrendes etwas (Kind, Kreisel), getirrel Geschwirre, engl. thirl thrill drillen, bohren, nd. tier-lier Gesang der Lerche, jedes Geklinge, Vb. tier-lieren, engl. tirra-lirra, ostfries. tirliren wie die Lerchen oder Schwalben singen oder trillern, und das im Ablaut stehende nd. türlüren, (= fr. turelure), sowie nd. nld. ostfries. trillen hin- und herschütteln, zittern, beben ( $=$ it. trillare), ostfries. trillern zittern, beben, klirren.

8 Vik wie vic haben eig. ein langes $\hat{\imath}$. 
zum mindesten unberechtigt ist. Ebenso wenig darf man ihm zustimmen, wenn er das altfr. guischet (pr. guisquet) aus jenem guichet durch Einschub eines $s$ erklärt. Diesem guischet liegt vielmehr ein zum ahd. wiskan, mhd. wischen mit etwas über etwas hinfahren, wischen, abwischen, abtrocknen, sich leicht und schnell bewegen, auch huschen, schlüpfen, (vgl. auch das amhd. int-wisken entwischen, neben ahd. int-wîchan entweichen, zurückweichen, nachgeben), gehöriges Wort zu Grunde, wie ahd. wisc, mnd. wisch, ostfries. wisk, das allerdings meist nur die Bedeutung „Wisch, Bündel“, im Ostfries. daneben aber „rasche Bewegung, Husch" etc. hat. Ebenso ist das nld. winket wohl nicht aus guichet abgeleitet, sondern aus einer $\mathrm{zu}$ ahd. winchan, mnld. wincken sich wenden, drehen, gehörigen Bildung.

Das afr. guiche und guige Band, it. guiggia scheint ähnlicher Herkunft zu sein wie guichet. Zwar möchte es Diez auf ein Wort des Kasseler Glossars windicas, den Pl. vom ahd. wintic (fascia, fasciola, Beinbinde, Strumpf) zurückführen, obwohl, wie er sagt, der im Franz. unübliche Ausfall des $n$ vor $d$ einen kleinen Schatten auf diese Deutung werfe.

Eine ähnliche Bedeutung wie in guiche finden wir im nhd. die wichel Tuch oder Band zum Wickeln der Kinder oder um etwas herum (s. Weigand, neben der wickel etwas Zusammengedrehtes, Zusammengewickeltes, ostfries. wikkel, ahd. wichili, pensum, manipulus), und im mhd. wickeln, mnld. wickelen, ostfries. wikkeln, wickeln, winden, schlingen, drehen. Daneben findet sich noch md. wîke, wieke, wicke Lampendocht aus Garn gedreht, gedrehte Charpie, spätahd. wieche Charpie (gl. herrad. 185a) wîh (gl. Trevir. 16, 15) Docht, denen im Nld. wiecke (elychnium, turunda, linamentum, pannus, lemniscus, = Band, Charpie etc.) zur Seite steht. Diese Bildungen erscheinen als Ableitungen aus einem anzusetzenden ahd. wicki wickja (vgl. an. vik rasche Drehung und Wendung) sowie mnld. wicken vibrare, librare, hin- und herbewegen, schütteln etc., hess. zvicken rasch und kräftig hin- und herbewegen, ahd. wîhôn, md. wiken tanzen, hüpfen, springen, mhd. wicken emporschnellen. Der Begriff „Binde, Band“ entwickelt sich wie beim an. veipr Kopftuch, Kopfbinde, got. vipja Kranz, Krone, die zu dem in ahd. wîfan windend, schwingend hin- und herbewegen, vorliegendem Stamm vip gehören.

Die Nebenformen fr.guige, it.guiggia würden sich als Bildungen aus einem neben dem germ. Stamme vik auftretenden Schwesterstamme vig erklären, der im ostfries. wiggen wiegen, schwingen, hin- und herbewegen, schaukeln, got. ga-vigan bewegen, schütteln, schwingen, ahd. wëgan und in der Form vîg im ahd. wîga Wiege, Gerät zum Hin- und Herbewegen, vorliegt.

Das oben genannte windicas, ahd. wintinc Beinbinde, Strumpf, vindinca, gehört $\mathrm{zu}$ dem Stamme vind, wie ahd. wintan windan winden, wickeln, as. windan (= it. ghindare, fr. guinder aufwinden) etc., ostfries. windsel winsel Windel, Wickelband. Das fr. bei Diez erwähnte guinche hingegen (Aye d'Av. p. 85) sowie das mdartl. s'aguincher sich schmücken, sind zum Stamme wink zu stellen. Vgl.

Zeits.chr. f. rom. Phil. XVIII. 
ahd. winch Wink, Wanken, ahd. winchan sich seitwärts bewegen, wanken, winken, sich von einer Seite zur andern neigen, wie man beim Schmücken thut, um $z u$ sehen, ob alles gut sitzt.

Guiper afr. überspinnen, wirken, guipure eine Art Spitzen, stellt Diez zunächst mit dem got. veipan bekränzen, mhd. wîfan, nhd. weifen zusammen, möchte es aber, da ihm die Bedeutung "vinden, windend bewegen oder drehen, schwingen" des mhd. Wortes entgangen $\mathrm{zu}$ sein scheint, lieber auf das ahd. wëban (wëpan) weben, zurückführen. Er vergleicht it. aggueffare beifügen, zu cem er bemerkt, es bedeute eigentlich "anweben", wie lat. adtexer?, und stamme vom ahd. wifan weben.

Diese Angaben leiden an einer gewissen Unklarheit. Zunächst ist ohne weiteres kein ahd. wifan mit kurzem $\check{z}$ nachzuweisen, bei Schade findet sich nur ein in dem ahd. Kompositum bervifin auftretendes st. Vb. wîfan, dessen langes $\hat{\imath}$ im Romanischen nur $i$ ergeben mulste, und von dem aggueffare selbst nicht stammen kann. Sodann stimmt das afr. guiper lautlich nicht $\mathrm{zu}$ dem ahd. wëban und ebenso wenig $\mathrm{zu}$ einem daraus für das Got. anzusetzenden viban. Wir werden uns somit an eine got.-fränk. dem ahd. wîfan entsprechende Form wie wîpan halten müssen, wie es schon Waltemath (ebenso wie er fr. gripper auf ein frk. ${ }^{*}$ gripan ${ }^{1}$ zurückführt) thut. Das ahd. bewîfen hatte, worauf Waltemath nicht aufmerksam macht, ursprünglich den Sinn „bewinden, umwinden, dann fesseln",2 wie das den Ablaut des Sg. Praet. von wîfan zeigende mhd. zweifen neben „schwingen“ auch „haspeln“ bedeutet. Man vgl. auch ahd. weif, waif was man um etwas windet, schlingt und wickelt, mhd. weif Umwindung, Herumgewundes (an. veipr Kopftuch, Kopfbinde, got. vaips Kranz, Krone), mhd. weife Garnwinde, Haspel, mhd. wifelen wifeln mit der Nadel einen Rifs stopfen, flicken, nhd. schweiz. schwäb. wifeln, verwifeln dass. Aus der Bedeutung „bewinden, umwinden, haspeln" etc. ergiebt sich die von „überspinnen, wirken“, und des weitern die von "Spitze", die auch in dem wohl wieder aus dem Fr. übernommenen mhd. (Lanz. 444I) zeeifier oder weifiere eine Art Spitzen, vorliegt.

In der lex Bajuv. und der lex. Ludov. wird ein wiffa, wifa „ein Merkzeichen, das einen verbotenen Weg anzeigt oder ein Grundstück zu betreten warnt" erwähnt. Es scheint dasselbe Wort $\mathrm{zu}$ sein wie zeifen ( 15 . Jahrh.) Merkzeichen von Grundstücken, nhd. schweiz. wîfe Warnungszeichen im See, hess. wîfstecken weifstecken (16. Jahrh.) Stecken mit Strohlehm umwickelt, nd. wîp Wisch von Stroh, womit man etwas abwischt oder zustopft, (strô-wîp etc.), holstein. hamburg. wîpe, altmärk. wîpen ein auf einer Stange errichteter Strohwisch, um einen verbotenen Weg zu zeigen.

Als Verbalbildungen treten daneben auf nhd. bair. ( 16 . Jahrh.) weifen Grenzmarken festsetzen, (vgl. auch nd. ditm. wipen ein Ziegel-

$1 \mathrm{Vgl}$. nd. Formen wie afries. grîpa, ostfries. grîpen neben gripen, mnld. grîpen, as. ags. grîpan, neben ahd. grîfan.

2 Vgl. bewif $(f)$ en condemnatus, miser. 
dach statt mit Kalk mit Stroh dicht machen, ags. vîpjan wischen, aengl. zuîpin wischen, abwischen) und ein dem oben genannten zoif(f)a entsprechendes langob. lat. wif(f)are guif(f)are etwas mit einem best. Merkzeichen versehen, durch ein aufgestecktes Zeichen (Strohwisch) die Besitznahme eines Grundstücks erklären (J. Grimm, Deutsche Rechtsaltert. 195 fg. 94I). Schade ist bei wif(f)are in Zweifel, ob es auf das in den vorgenannten Bildungen $z u$ Tage tretende schwache Vb. wîfjan oder auf das oben genannte starke ahd. wîfan zurückzuführen sei. Die lat. Endung are würde eher auf Uebereinstimmung mit wîfan deuten, zu dem auch das fr. giffer ${ }^{1}$ ein Haus konfiscieren, welches Diez zu aggueffare stellen möchte, lautlich stimmt. Aber schon Grimm hat a. a. O. 94I das oben besprochene sbst. wiff(f)a, für das Schade auf Grund der späteren bair. Form mit $e i$ langes $\hat{\imath}$ in Anspruch nimmt, mit dem got. vipja Kranz, Krone, für identisch erklärt, zu dem es auch begrifflich stimmt. Dazu kommt, dafs Schades Behauptung, wif(f)a müsse ein langes $i$ haben, nicht stichhaltig ist, da sich, was ihm entgangen, im Deutschen auch sonst ähnliche Bildungen mit $\bar{i}$ nachweisen lassen, die neben jenen mit $\hat{\imath}$ nebenherlaufen, so im Mnd. Ostfries. neben wip auch wipe, im Mnld. sogar allein wip (wyp), im Schwed. vippa Büschel etc. Wir dürfen also auch für das langob. Vb. wif(f)an ebenso gut ein kurzes $i^{2}$ ansetzen, um so mehr, als das auf it. Boden erwachsene aggueffare ein solches unbedingt fordert. Das letztere könnte somit als eine unmittelbar aus einem langob. lat. wiff(f)a abgeleitete Verbalbildung angesehen werden oder direkt von wif(f)an stammen.

$1 \mathrm{Vgl.} \mathrm{zum} \mathrm{Anlaut} \mathrm{afr.} \mathrm{gerpir,} \mathrm{gile,} \mathrm{nfr.} \mathrm{givre,} \mathrm{gêpe} \mathrm{(in} \mathrm{Berry),} \mathrm{Gérard}$ = Guérard etc.

$2 \mathrm{Vgl}$. auch mhd. wifeln flicken etc., das ein älteres ziif:n voraussetzt, sowic aift feiner liaden, ahd. wiftjan texere etc.

ThEODOR BRAUNe.

\section{Verzeichnis der auf den Seiten 5I3-53I behandelten Etymologien.}

aggueffare it. S. 530/31. bague fr. S. 515 .

baud fr. S. 519.

cahute cajute fr. S. $520 \mathrm{ff}$. chiffe chippe fr. etc.

S. 522 .

chirlar sp. pg. S. 528 .

chiurlare it. S. 528.

fade fr. S. 514 .

gaignon afr. S. 517 .

garawa ahd., mhd. garbe

S. 526 .

garbo ven. trient. S. 525 . goffo it., fr. goffe S. 524 . gorra it. sp. pg. S. 523 . gufo it. S. 525 . guoffola ncap. S. 526,27 . guiche afr. S. 529 . guichet fr. S. 528 . guige afr. S. 529 . guiper afr. S. 530 . guischet afr. S. 529 . huge huche fr. S. 513. huivar pg. S. 527. huler afr. S. 527. hurler fr. S. 527. juc, jucher fr. S. 5 I 3 . mests afr. S. 514 . mets fr. S. 514 . metz afr. S. 514 . stamberga it. S. 5I9. trouver fr., it. trovare etc. S. 516/17. udolar pr. S. 527. uler afr. S. 527 . urlare it. S. 527 . viquet wiket afr. S. 528 . zinzilulare barbar. lat.

S. 527 Anm. 1 . zirlare it. S. 528 . 